\title{
L. NEMETI
}

\section{Sur le problème d'ordonnancement dans la fabrication en série}

Revue française d'automatique, d'informatique et de recherche opérationnelle. Recherche opérationnelle, tome 2, no V2 (1968), p. $47-60$.

$<$ http://www.numdam.org/item?id=RO_1968_2_2_47_0>

(C) AFCET, 1968, tous droits réservés.

L'accès aux archives de la revue « Revue française d'automatique, d'informatique et de recherche opérationnelle. Recherche opérationnelle » implique l'accord avec les conditions générales d'utilisation (http://www.numdam.org/ legal.php). Toute utilisation commerciale ou impression systématique est constitutive d'une infraction pénale. Toute copie ou impression de ce fichier doit contenir la présente mention de copyright.

\section{Numdam}

Article numérisé dans le cadre du programme

Numérisation de documents anciens mathématiques

http://www.numdam.org/ 
R.I.R.O.

$\left\{2^{e}\right.$ année, No 11,1968, p. 47-60

\title{
SUR LE PROBLEME D'ORDONNANGEMENT DANS LA FABRICATION EN SERIE
}

\author{
par L. Nemeti (1)
}

\begin{abstract}
Résumé. - On donne dans cet article un modèle de l'ordonnancement dans la fabrication en série. Celui-ci représente un problème de programmation avec conditions logiques, à résoudre par l'algorithme de $F$. Radó. Après une présentation très succincte de cette méthode, on donne un procédé de résolution des problèmes nommés fondamentaux du modèle. Ce procédé est une généralisation et amélioration d'un algorithme de $R$. Cruon et $P h$. Hervé.
\end{abstract}

1. La fabrication en série est caractérisée par le fait que les tâches s'en répètent périodiquement. Dans les modèles usuels d'ordonnancement, c'est la durée totale de la fabrication qui doit être minimisée. Par contre, au cas de la fabrication en série, on util sera une autre fonction économique : ce sera la longueur maximale de la période de fonctionnement de chaque machine de l'atelier. MM. R. Cruon et Ph. Hervé [1] ont été les premiers à souligner cette circonstance.

Dans ce qui suit nous construirons un modèle de ce genre de fabrication et en indiquerons une méthode de résolution qui consiste en une généralisation et amélioration du procédé indiqué dans l'ouvrage [1]. Mentionnons tout d'abord que l'auteur a indiqué un modèle d'ordonnancement [2] pour le cas usuel qui constituait un problème de programmation linéaire à conditions logiques. Pour ces problèmes on peut utiliser l'algorithme de F. Radó [3], [4], apparenté aux méthodes "Branch et Bound " et "S.E.P.". Le modèle qui suit, constitue aussi un problème de ce genre.

Supposons qu'on ait $m$ machines à sa disposition et qu'on doive exécuter périodiquement $n$ tâches. Les machines seront numérotées de 1 à $m$ et les tâches de 1 à $n$. Désignons par $M=\{1, \ldots, m\}$, l'ensemble des nombres représentant les machines et par $N=\{1, \ldots, n\}$ l'ensemble des nombres représentant les tâches. Nous dirons simplement

(1) Institut de Calcul à Cluj de l'Académie de la République Socialiste de Roumanie. 
que $M$ est l'ensemble des machines et $N$ celui des tâches. L'ensemble $N$ admet une partition déterminée par les prescriptions technologiques :

$$
N=\bigcup_{k \in M} N_{k}
$$

où $N_{k}$ est le sous-ensemble des tâches qui sont exécutées sur la machine $k$. De même, ces prescriptions déterminent l'ensemble $H \subset N \times N$ ayant pour éléments les couples de tâches entre lesquelles il y a une relation de succession technologique, c'est-à-dire la relation $(i, j) \in H$ indique le fait que la tâche $j$ ne peut être commencée qu'après l'achèvement de la tâche $i$. On donne aussi la durée $t_{i}$ d'exécution de la tâche $i$ et on désigne par $x_{i}$ le moment (inconnu) du commencement de celle-ci.

Nous introduisons encore l'ensemble

$$
\bar{J}=\left\{(i, j) \mid i, j \in N_{k} ; k \in M\right\}=\bigsqcup_{k \in M}\left(N_{k} \times N_{k}\right)
$$

contenant les couples de tâches qui s'exécutent sur la même machine (on a $i \in N \Rightarrow(i, i) \in \bar{J}$ ) et l'ensemble

$$
J=\{(i, j) \mid i<j\} \cap \bar{J} .
$$

La durée (inconnue) de la période de fabrication sera désignée par $\varphi$. Les inconnues doivent vérifier les conditions

$$
\begin{cases}x_{j}-x_{i} \geqslant t_{i}, & (i, j) \in H \\ x_{j}-x_{i} \geqslant t_{i} \vee x_{i}-x_{j} \geqslant t_{j}, & (i, j) \in J \\ x_{j}-x_{i} \geqslant t_{i}-\varphi, & (i, j) \in \bar{J} \\ f=\varphi: \text { minimum. } & \end{cases}
$$

La deuxième condition s'appellera celle de la non-interférence et la troisième celle de la période.

Nous avons mentionné que ce problème de programmation à conditions logiques peut être résolu par l'algorithme de F. Radó. Cet algorithme prescrit la résolution d'une suite de problèmes de programmation ordinaire appelés problèmes fondamentaux. Ceux-ci sont constitués par les conditions ordinaires du problème et par le premier ou second terme d'une partie des disjonctions du problème. Par suite, un problème fondamental sera de la forme,

$$
\left\{\begin{array}{l}
x_{j}-x_{i} \geqslant t_{i}, \quad(i, j) \in L \quad(H \subset L) \\
x_{j}-x_{i} \geqslant t_{i}-\vartheta, \quad(i, j) \in \bar{J} \\
\varphi: \text { minimum }
\end{array}\right.
$$


2. Nous allons maintenant développer la méthode de résolution du problème posé par (1.5). Nous écrivons sous une forme plus générale :

$$
\begin{array}{ll}
x_{j}-x_{i} \geqslant a_{i j}, & (i, j) \in A \subset N \times N \\
x_{j}-x_{i} \geqslant a_{i}-\varphi, & (i, j) \in V \subset N \times N \\
\nu: \text { minimum } &
\end{array}
$$

avec les nombres réels $a_{i j}$ et $a_{i}$ et les ensembles $N, A, V$ donnés, les inconnues étant $\varphi$ et $x_{i}, i \in N$.

Il est évident que la valeur minimale cherchée de $\varphi$ est égale au plus petit nombre $\varphi$ pour lequel le système constitué par les conditions (2.1) et (2.2) est compatible.

On pourrait appeler ce problème un problème de quasi-potentiel. Il semblerait qu'il est au fond identique à celui traité dans [1]. En réalité, il y a des différences essentielles, notre problème étant une généralisation du modèle de [1]. Pour formuler les hypothèses que nous supposerons remplies, nous attachons d'abord à notre problème le graphe

$$
G=[N, A]
$$

dont l'arc $(i, j) \in A$ est doté de la "longueur " $a_{i j}$. Soient $i$ et $j$ deux sommets liés par un chemin dans le graphe $G$ et soit $m_{i j}$ la longueur du plus long chemin de $i$ à $j$. Soit

$$
G^{\prime}=[N, U]
$$

le graphe qui est la fermeture transitive de $G$, c'est-à-dire si les sommets $i$ et $j$ sont liés par un chemin dans $G$, alors ils sont liés par un arc dans $G^{\prime}$. La longueur de l'arc $(i, j)$ dans $G^{\prime}$ est précisément $m_{i j}$. Nous introduisons ensuite le graphe

$$
G^{\prime \prime}=[N, U \cup V],
$$

l'arc $(i, j)$ ayant la longueur

$$
\begin{array}{lll}
m_{i j}, & \text { si } & (i, j) \in U \\
a_{i}-\varphi, & \text { si } & (i, j) \in V^{(1)}
\end{array}
$$

Les arcs éléments de $V$ seront nommés des arcș "en $\varphi$ ".

Le graphe $G^{\prime}$ admet les sous-graphes

$$
G_{r}=\left[N_{r}, U_{r}\right], r \in M \quad\left(U_{r}=U \cap\left(N_{r} \times N_{r}\right)\right),
$$

où $M$ et $N=\bigcup_{r \in M} N_{r}$ ont été définis au paragraphe 1.

(1) Si les ensembles $U$ et $V$ admettent l'élément commun $(i, j)$, alors il y aura deux arcs $(i, j)$, l'un de longueur $m_{i j}$, l'autre de $a_{i}-\varphi$. 
Nos hypothèses sont les suivantes :

a) le système (2.1) est compatible,

b) l'ensemble $V$ admet la partition

$$
V=\bigcup_{r \in M} v_{r}
$$

avec

$$
V_{r}=N_{r} \times N_{r}
$$

c) Soit $(i, j) \in U_{r}$. On a

$$
m_{i j} \geqslant 0
$$

et

$$
m_{i j} \geqslant a_{i}-a_{j} .
$$

d) Il n'y a pas de sommets isolés dans $G$, respectivement dans $G^{\prime \prime}$. Les hypothèses dans [1] sont plus restrictives. On y suppose (outre les hypothèses $a, b, d)$ :

a) $a_{i} \geqslant 0$.

B) Dans un sous-graphe $G_{r}$, chaque sommet possède au plus un précêdent et au plus un suivant, c'est-à-dire que l'ensemble $U_{r}$ détermine un ordre dans $N_{r}$ tandis que, dans nos hypothèses, $N_{r}$ n'est que partiellement ordonné.

Soit maintenant $N_{r}^{\prime}$ une base et $N_{r}^{\prime \prime}$ une anti-base de $G_{r}$. Notons

$$
\left\{\begin{array}{l}
N_{r}^{0}=N_{r}^{\prime} \cup N_{r}^{\prime \prime} \\
N^{\prime}=\bigcup_{r \in M} N_{r}^{\prime} \\
N^{\prime \prime}=\bigcup_{r \in M} N_{r}^{\prime \prime} \\
N^{0}=N^{\prime} \cup N^{\prime \prime}=\bigcup_{r \in M} N_{r}^{0}
\end{array}\right.
$$

puis

$$
\left\{\begin{array}{l}
V_{r}^{0}=N_{r}^{0} \times N_{r}^{0} \\
V^{0}=V \cap\left(N^{0} \times N^{0}\right)=\bigcup_{r \in M} V_{r}^{0} \\
\bar{V}=V \cap\left(N^{\prime} \times N^{\prime \prime}\right) \subset V^{0} \\
U^{0}=U \cap\left(N^{0} \times N^{0}\right) .
\end{array}\right.
$$


L'ensemble $\bar{V}$ contient tous les arcs "en $\varphi$ " de la réunion $N^{\prime}$ des bases à la réunion $N^{\prime \prime}$ des anti-bases. Par ces ensembles, nous définissons le sous-graphe de $G^{\prime \prime}$ :

$$
G^{0}=\left[N^{0}, U^{0} \cup V^{0}\right],
$$

où n'interviennent que les sommets $N^{\circ}$ et le graphe partiel

$$
\bar{G}=\left[N^{0}, U^{0} \cup \bar{V}\right] \text {. }
$$

Après ces préliminaires, remarquons que le système (2.1) équivaut au système

$$
x_{j}-x_{i} \geqslant m_{i j}, \quad(i, j) \in U .
$$

Considérons le système d'inégalités

$$
\begin{aligned}
& x_{j}-x_{i} \geqslant m_{i j}, \quad(i, j) \in U^{0} \\
& x_{j}-x_{i} \geqslant a_{i}-\varphi, \quad(i, j) \in V^{0}
\end{aligned}
$$

attaché au graphe $G^{\circ}$.

Nous démontrerons le

Théorème. - On se donne le système d'inégalités $S$ défini par les formules $\left(2.1\right.$ a) et $(2.2)$ et le système $S^{0}$ défini par (2.9) et (2.10). Soit $\hat{v}$ le plus petit nombre $\varphi$ pour lequel le système $S$ est encore compatible et $\omega^{0}$ le même nombre pour le système $S^{\circ}$. On a :

$$
\hat{\varphi}=\varphi^{0} \text {. }
$$

Démonstration. - Le théorème résulte immédiatement des deux propositions suivantes :

a) Si le système $S$ est compatible pour le nombre $\varphi$, alors le système $S^{0}$ est aussi compatible pour ce nombre. C'est évident.

b) Si le système $S^{0}$ est compatible pour le nombre $\varphi$, alors le système $S$ est aussi compatible pour ce nombre.

Soit le vecteur $X=\left\{x_{i} \mid i \in N\right\}$ la solution des conditions $(2.1 a)$ obtenue par

$$
x_{i}=\max _{s \in N a}\left(k_{s}+m_{s i}\right), \quad i \in N
$$

où $N_{a}$ est une anti-base du graphe $G^{\prime}$ et les constantes $k_{s}, s \in N_{a}$ sont arbitraires. Cette formule est une généralisation de la solution de B. Roy [5] d'un problème de potentiels :

$$
x_{i}=\max _{s \in N} m_{s i}, \quad i \in N .
$$


Le vecteur $X^{\mathrm{o}}=\left\{x_{i} \mid i \in N^{\circ}\right\} \subset X$ est évidemment une solution des inégalités (2.9). Nous supposerons la valeur de $\varphi$ telle que $\varphi$ et $X^{0}$ vérifient les conditions (2.10). Nous montrerons qu'en ce cas $v$ et $X$ vérifient les conditions (2.2) ce qui démontrera la proposition $b$ ).

Considérons deux sommets $i, j \in N_{r}$, où $r$ est un élément de $M$. Soit $\alpha$ un sommet de $N_{r}^{\prime \prime}$ pour lequel on a

$$
x_{i}=x_{\alpha}+m_{\alpha i}
$$

et soit $\gamma$ un sommet de $N_{r}^{\prime}$ pour lequel le nombre $m_{i \gamma}$ est défini. On a alors

$$
x_{\gamma} \geqslant x_{i}+m_{i \gamma}
$$

D'une manière analogue, nous écrirons : $\beta \in N_{\text {r }}^{\prime \prime}$

$$
x_{j}=x_{\beta}+m_{\beta j}
$$

et $\delta \in N_{r}^{\prime}$

$$
x_{\delta} \geqslant x_{j}+m_{j \delta} .
$$

En vertu de la prémisse de la proposition $b$ ), on a

$$
\nu \geqslant x_{\gamma}+a_{\gamma}-x_{\beta}
$$

et

$$
\varphi \geqslant x_{s}+a_{\delta}-x_{\alpha} .
$$

En tenant compte de (2.14) et (2.15), nous obtenons de (2.17):

$$
\vartheta \geqslant x_{i}-x_{j}+m_{i \gamma}+a_{\gamma}+m_{\beta j}
$$

et, similairement

$$
\varphi \geqslant x_{j}-x_{i}+m_{j \delta}+a_{\delta}+m_{\alpha i}
$$

On en déduit en utilisant les propriétés (2.5) et (2.6) :

$$
\begin{aligned}
& \vartheta \geqslant x_{i}-x_{j}+a_{i} \\
& \nu \geqslant x_{j}-x_{i}+a_{j} .
\end{aligned}
$$

Puisque ces inégalités ont lieu pour tout $r \in M$, nous sommes arrivés aux relations (2.2). La proposition $b$ ) est démontrée et, avec elle, le théorème aussi.

En vertu du théorème, nous pouvons déterminer la valeur de $\hat{\varphi} \mathrm{du}$ système $S^{o}$ ce qui raccourcit les calculs. Mais nous pouvons continuer les simplifications. 
Nous affirmons d'abord que dans le graphe $G^{\circ}=\left[N^{\circ}, U^{\circ} \cup V^{\circ}\right]$ on a :

$$
\left(i \in N_{r}^{\prime} j \in N_{r}^{\prime \prime}\right) \Rightarrow(i, j) \notin U^{0}, \quad r \in M \text {. }
$$

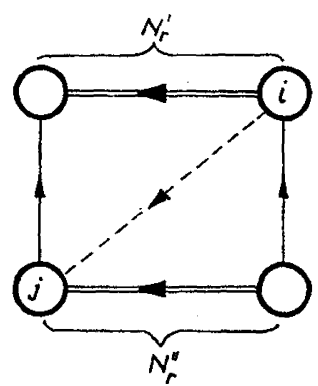

a)

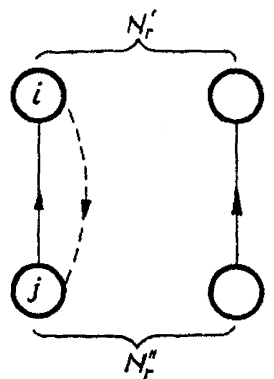

b)

Figure 1

En effet, au cas contraire il résulterait (voir fig. 1) ou bien qu'il y a un chemin entre deux sommets de la base et de l'anti-base (marquée par une ligne double dans la figure) ce qui contredit la définition de la base respectivement de l'anti-base, ou bien que les sommets $i$ et $j$ sont reliés dans les deux sens, c'est-à-dire qu'il existe un circuit d'une longueur positive [voir la propriété (2.5)] ce qui contredit l'hypothèse $a$ ).

En deuxième lieu, on a

$$
v \geqslant a_{i}, \quad i \in N
$$

ce qui est immédiat, si l'on pose $j=i$ dans la condition (2.2).

Une fois en possession de ces résultats, nous montrerons que $(A)$ pour déterminer la valeur $v^{\circ}$ il suffit de considérer parmi les arcs $(i, j) \in V^{\circ}$ seulement les arcs avec

$$
i \in N^{\prime}, j \in N^{\prime \prime} \text {. }
$$

Dans ce but nous démontrerons que les autres arcs "en $\theta$ n'ont aucune influence sur la valeur de $\nu^{\circ}$. Nous rappelons que la valeur de $\varphi^{\circ}$ résulte de la condition que tout circuit (en $G^{\circ}$ ) contenant des arcs "en $\varphi$ » a une longueur négative (ou nulle). Supposons qu'un tel circuit $\gamma$ contienne des $\operatorname{arcs}(i, j)$ des quatre $\operatorname{arcs}$ "en 0$)$ :

$$
\begin{aligned}
& \text { 1) } \quad i \in N^{\prime}, j \in N^{\prime \prime} \\
& \text { 2) } \quad i \in N^{\prime}, j \in N^{\prime} \\
& \text { 3) } \quad i \in N^{\prime \prime}, j \in N^{\prime \prime} \\
& \text { 4) } \quad i \in N^{\prime \prime}, j \in N^{\prime}
\end{aligned}
$$

de longueur $a_{i}-\nu$. Nous montrerons qu'il y a dans $G^{\circ}$ un autre circuit $\gamma^{\prime}$ contenant au plus autant d'arcs "en $\gamma$ " que $\gamma$, mais tous de la catégorie 1 et ayant une longueur plus grande que $\gamma$. Alors la valeur de $\varphi$, déduite de $\gamma^{\prime}$ sera supérieure à celle déduite de $\gamma$ ce qui prouvera la proposition $(A)$. 
Considérons d'abord un arc $(i, j)$, de la catégorie 2 . Il va de soi que les sommets $i$ et $j$ se trouveront tous les deux dans le même sous-graphe $G_{r}$ puisqu'autrement il n'y aurait pas un arc "en $\varphi$ " qui les joigne. Il y a un sommet $k \in N_{r}^{\prime \prime}$ tel qu'il existe un chemin de $k$ à $j$ de la longueur $m_{k j}$. D'autre part il y a un arc "en $(\eta)(i, k)$ (de la catégorie 1) puisque les deux sommets appartiennent à $G_{r}$. Cet arc, de même que l'arc $(i, j)$, est de longueur $a_{i}-\varphi$. Nous remplaçons l'arc "en $\varphi$ " $(i, j)$ par le chemin $[i, k, j]$ de longueur $a_{i}-\varphi+m_{k j}$, plus grande que la longueur de $(i, j)$ (voir la propriété (2.5)).

On peut éliminer les arcs de la catégorie 3 par un raisonnement à peu près identique.

Dans la catégorie 4 nous distinguerons plusieurs cas :

Premier cas. La base $N_{r}^{\prime}$ et l'anti-base $N_{r}^{\prime \prime}$ contiennent en plus de $j$ resp. $i$, au moins un sommet $l$ resp. $k$ et il n'y a pas de chemins en $U$ de $i$ à $j$. Alors il y a des chemins (en $U$ ) de $i$ à $l$ (longueur maximale $m_{i l}$ ) et de $k$ à $j$ (longueur maximale $m_{k j}$; voir fig. 2).

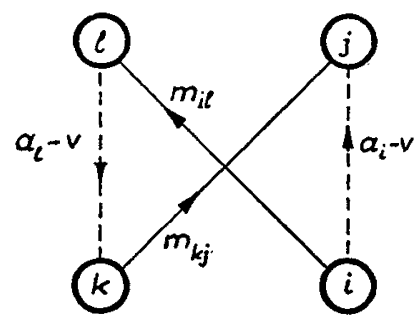

Figure 2

Nous remplą̧ons l'are $(i, j)$ de longueur $a_{i}-\varphi$ par le chemin $[i, l, k, j]$ de longueur $m_{i j}+a_{l}-\varphi+m_{k j}$. Cette longueur est, en vertu de la propriété (2.6) supérieure à $a_{i}-\varphi+m_{k j}$ qui est, à son tour, plus grande que $a_{i}-\varphi$, la longueur de l'arc $(i, j)$.

Second cas. La base $N_{r}^{\prime}$ contient le seul élément $j$ (fig. 3 ) et il n'y a pas de chemins de $i$ à $j$ en $U$.

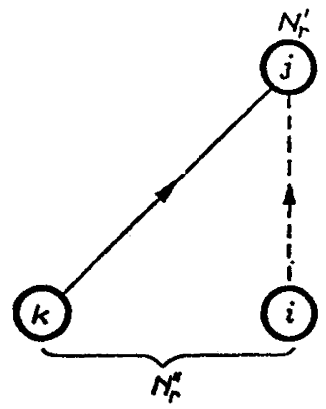

Figure 3 
Alors le sommet $i$ est isolé dans le graphe $G_{r}$ (dans $G^{\prime}$ il n'est pas isolé !) et nous pouvons le considérer au même titre comme faisant partie de $N_{r}^{\prime}$. Par cela, l'arc $(i, j)$ entre dans la catégorie 2, déjà traitée.

Troisième cas. L'anti-base $N_{r}^{\prime \prime}$ contient le seul sommet $i$ et il n'y a pas de chemins $[i, j]$ en $U$. Ce cas est évidemment impossible.

Quatrième cas. Il y a des chemins $[i, j]$ en $U$ (longueur maximale $m_{i j}$ ). Nous remplaçons alors l'arc $(i, j)$ " en $v$ ", de longueur $a_{i}-v$ au plus nulle [voir $(2.22)$ par le chemin $[i, j] \in U$, de longueur $m_{i j}$ positive.

En procédant ainsi avec tous les ares " en $\varphi$ " du circuit $\gamma$ nous arrivons au circuit $\gamma^{\prime}$, ayant les propriétés énoncées plus haut. La proposition $(A)$ est démontrée.

En conclusion, il suffit de résoudre le système

$$
\begin{array}{ll}
x_{j}-x_{i} \geqslant m_{i j}, & (i, j) \in U^{0} \\
x_{j}-x_{i} \geqslant a_{i}-\varphi, & (i, j) \in \bar{V}
\end{array}
$$

avec un $\varphi$ minimal. A ce système est attaché le graphe $\bar{G}=\left[N^{0}, U^{0} \cup \bar{V}\right]$.

Le graphe $\bar{G}$ est la "somme " de $m$ sous-graphes $\bar{G}_{r}, r \in M$ et chacun d'eux est une juxtaposition de deux graphes simples : les sommets en sont positionnés en deux sous-ensembles $N_{r}^{\prime}$ et $N_{r}^{\prime \prime}$, les ares étant partitionnés eux aussi : les arcs $(i, j) \in U^{0}$ du graphe $\bar{G}_{r}$ sont tous incidents à $N_{r}^{\prime \prime}$ vers l'extérieur et à $N_{r}^{\prime}$ vers l'intérieur, les $\operatorname{arcs}(i, j) \in \bar{V}$ du graphe $\bar{G}_{r}$ sont tous incidents à $N_{r}^{\prime}$ vers l'extérieur et à $N_{r}^{\prime \prime}$ vers l'intérieur. Les sous-graphes $\bar{G}_{r}$ sont liés entre eux par des arcs éléments de $U^{\circ}$.

3. Afin de déterminer la valeur minimale de $\varphi(=\bar{\rho}=\hat{\varphi})$ pour laquelle le graphe $\bar{G}$ ne présente aucun circuit de longueur positive, on étudiera, l'un après l'autre, les circuits de $\bar{G}$ contenant $1,2, \ldots$ arcs " en $\varphi$ ".

On désignera par $\varphi_{p}$ la plus petite valeur de $\varphi$ pour laquelle il n'y a aucun circuit de $\bar{G}$ de longueur positive et contenant $p$ ares "en $\theta$ ". Nous avons, évidemment

$$
\hat{\vartheta}=\max \left(\varphi_{1}, \varphi_{2}, \ldots, \varphi_{v}\right), \quad \nu=\left|N^{0}\right|,
$$

étant le nombre des sommets en $\bar{G}$,

a) Circuits contenant un seul arc "en $\varphi$ ». Un tel circuit contient les sommets $[\alpha, \beta, \alpha], \alpha \in N_{r}^{\prime} \beta \in N_{r}^{\prime \prime}, r \in M$. La longueur de l'arc $(\alpha, \beta)$ est $a_{\alpha}-\varphi$, celle de l'arc $(\beta, \alpha)$ est $m_{\beta \alpha}$. On aura par conséquent

$$
\varphi_{1} \geqslant a_{\alpha}+m_{\beta \alpha},
$$


c'est-à-dire

$$
\rho_{1}=\max _{\substack{\alpha \in N^{\prime} \\ \beta \in N^{\prime} \\(\beta, \alpha) \in U^{\circ}}}\left(a_{\alpha}+m_{\beta x}\right) .
$$

b) Circuits contenant $p$ arcs "en $p$ ». Désignons ces arcs par

avec

$$
\left(\alpha_{1}, \beta_{1}\right),\left(\alpha_{2}, \beta_{2}\right), \ldots,\left(\alpha_{s}, \beta_{s}\right), \ldots,\left(\alpha_{p}, \beta_{p}\right)
$$

$$
\alpha_{s} \in N^{\prime}, \beta_{s} \in N^{\prime \prime}, \quad s=1,2, \ldots, p \text {. }
$$

Le circuit qui parcourt ces arcs dans l'ordre sus-indiqué est

$$
\Gamma=\left[\left(\alpha_{1}, \beta_{1}\right),\left(\beta_{1}, \alpha_{2}\right),\left(\alpha_{2}, \beta_{2}\right), \ldots,\left(\beta_{p-1}, \alpha_{p}\right),\left(\alpha_{p}, \beta_{p}\right),\left(\beta_{p}, \alpha_{1}\right)\right]
$$

où les $\operatorname{arcs}\left(\beta_{s}, \alpha_{s+1}\right)$ appartiennent à $U^{0}$ et les $\operatorname{arcs}\left(\alpha_{s}, \beta_{s}\right)$ à $\bar{V}$.

La longueur de ce circuit est

$$
a_{\alpha_{1}}+m_{\beta_{1} \alpha_{2}}+a_{\alpha_{2}}+m_{\beta_{2} \alpha_{3}}+\ldots+a_{\alpha_{p}}+m_{\beta_{p} \alpha_{1}}-p v
$$

d'où la condition

$$
p \varphi_{p} \geqslant d_{\alpha_{1} \alpha_{2}}+d_{\alpha_{2} \alpha_{3}}+\ldots+d_{\alpha_{p} \alpha_{1}} .
$$

On a noté ici

$$
d_{\alpha \beta}=a_{\alpha}+\max _{\gamma \in P \alpha(\beta)} m_{\gamma \beta}, \quad \alpha, \beta \in N^{\prime},
$$

où $P_{\alpha}(\beta)$ est l'ensemble des précédents du sommet $\beta$ (en $\bar{G}$ ) adjacents à $\beta$ par un arc de $U^{0}$ qui appartiennent au même ensembìe $N_{r}^{0}$ que le sommet $\alpha$. Si $P_{\alpha}(\beta)$ est vide, alors le nombre $d_{\alpha \beta}$ n'est pas défini et n'entre pas dans la composition de la formule (3.3). La formule (3.4) fournit pour $p=1$

$$
v_{1}=\max _{\alpha \in N^{\circ}} d_{\alpha \alpha}
$$

identique avec $(3.2)\left({ }^{1}\right)$.

La simplification des calculs peut être poussée encore plus loin. Introduisons à cette fin le graphe

$$
G^{*}=\left[N^{\prime}, U^{\prime}\right]
$$

déduit du graphe $\bar{G}$, avec l'ensemble d'arcs

$$
U^{\prime}=\left\{(i, j) \mid j \in N^{\prime},(h, j) \in U^{0}, h \in N_{r}^{0}, i \in N_{r}^{\prime}\right\}
$$

(1) Jusqu'ici notre procédure est au fond identique avec celle de R. Cruon et $\mathrm{Ph}$. Hervé. 
c'est-à-dire un couple des sommets $(i, j)$ avec $i, j \in N^{\prime}$ est un arc élément de $U^{\prime}$ si et seulement s'il y a un chemin en $\vec{G}$ du sommet $i$ au sommet $j$ composé de deux arcs : le premier $(i, h)$ étant "en $v$ ", le deuxième $(h, j)$ sans $\varphi$ (fig. 4). On attribue à l'arc $(i, j) \in U^{\prime}$ la longueur $d_{i j}(3.4)\left({ }^{1}\right)$. Remarquons que tout sommet $i \in N^{\prime}$ possède aussi une boucle de longueur $d_{i i}$.

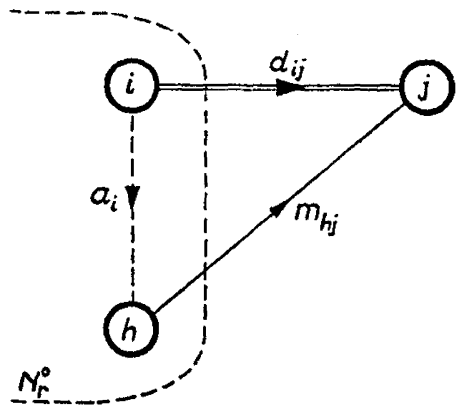

Figure 4

A chaque couple d'arcs $\left(\alpha_{s}, \beta_{s}\right),\left(\beta_{s}, \alpha_{s+1}\right)$ du circuit $\Gamma$ correspond un arc $\left(\alpha_{s}, \alpha_{s+1}\right)$ dans le graphe $G^{*}$. Au circuit $\Gamma$ d'ordre $2 p$ en $\bar{G}$ correspond un circuit $\Gamma^{*}$ d'ordre $p$ en $G^{*}$, d'une longueur positive

$$
d_{\alpha_{1} \alpha_{2}}+d_{\alpha_{2} \alpha_{3}}+\ldots+d_{\alpha_{p} \alpha_{3}}
$$

qu'on peut utiliser dans la formule (3.3).

La méthode de calculer $\hat{v}$ sera donc la suivante : on détermine dans le graphe $G^{*}$ - contenant $\nu^{\prime}=\left|N^{\prime}\right|$ sommets - tous les circuits d'ordre

$$
p=1,2, \ldots, \nu^{\prime} \text {; }
$$

tous ont une longueur positive. Soit $\lambda_{p}$ la longueur du plus long circuit d'ordre $p$. On a

$$
\hat{\vartheta}=\max \left(\lambda_{1}, \frac{\lambda_{2}}{2}, \ldots, \frac{\lambda \nu^{\prime}}{v^{\prime}}\right) .
$$

La détermination des circuits de divers ordres dans le graphe $G^{*}$ peut être facilitée à l'aide des matrices à éléments définis sur l'ensemble $\hat{R}=R \cup\{\theta\}(R:$ l'ensemble des nombres réels, $\theta$ interprété comme $-\infty$ ) introduites par $\mathrm{R}$. Cruon et $\mathrm{Ph}$. Hervé [1]. La procédure est la suivante :

Premier pas. On détermine la matrice $\mathfrak{D}=\left(\delta_{i j}\right)$ correspondant au graphe $G^{*}=\left[N^{\prime}, U^{\prime}\right]$ en posant

$$
\delta_{i j}=\left\{\begin{array}{lll}
d_{i j}, & \text { si } & (i, j) \in U^{\prime} \\
\theta, & \text { si } & (i, j) \notin U^{\prime}
\end{array}\right.
$$

(1) Le sommet $j$ peut être aussi bien un élément de $N^{\circ}$ qu'en dehors de celui-ci. 
$2^{\text {e }}$ pas. On calcule les puissances

$$
\mathfrak{D}, \mathfrak{D}^{*(2)}, \ldots, \mathfrak{D}^{*\left(v^{\prime}\right)}
$$

3e pas. A chaque puissance $\mathfrak{D}^{*(p)}$, on détermine la valeur $\Lambda_{p}(>0)$ de l'élément le plus grand de la diagonale principale.

$4^{\mathrm{e}}$ pas. On a

$$
\hat{\vartheta}=\max \left(\Lambda_{1}, \frac{\Lambda_{2}}{2}, \ldots, \frac{\Lambda_{\mathrm{v}^{\prime}}}{v^{\prime}}\right) .
$$

Nous devons démontrer que les formules (3.7) et (3.8) sont équivalentes. En effet, le nombre $\Lambda_{p}$ est égal (voir [1]) à la longueur maximale des circuits d'ordre au plus $p$ dans le graphe $G^{*}$, tandis que le nombre $\lambda_{p}$ indique la longueur maximale des circuits d'ordre $p$. Désignons par

a)

$$
\left\{\begin{array}{l}
z_{p}=\max \left(\lambda_{1}, \frac{\lambda_{2}}{2}, \ldots, \frac{\lambda_{p}}{p}\right) \\
Z_{p}=\max \left(\Lambda_{1}, \frac{\Lambda_{2}}{2}, \ldots, \frac{\Lambda_{p}}{p}\right) .
\end{array}\right.
$$

Nous démontrerons qu'on a

$$
z_{p}=Z_{p}, \quad p=1,2, \ldots
$$

Les relations suivantes sont évidentes :

c)

$$
\begin{gathered}
\Lambda_{p} \geqslant \lambda_{p} \\
\Lambda_{1}=\lambda_{1} \quad\left(Z_{1}=z_{1}\right) \\
\Lambda_{p+1} \geqslant \Lambda_{p} \\
\left(\Lambda_{p}>\lambda_{p}\right) \Rightarrow\left(\Lambda_{p}=\lambda_{p-1}\right) .
\end{gathered}
$$$$
\text { d) }
$$$$
\text { e) }
$$

Supposons qu'il y ait lieu

g)

$$
z_{p-1}=Z_{p-1}=\zeta
$$

On a alors

$$
\text { h) } \quad z_{p}=\max \left(\zeta, \frac{\lambda_{p}}{p}\right), \quad Z_{p}=\max \left(\zeta, \frac{\Lambda_{p}}{p}\right) \text {. }
$$

Nous distinguerons les cas suivants

$\alpha) \Lambda_{p}=\lambda_{p}$. Alors il résulte de $(h)$ que la formule $(b)$ est vraie.

B) $\Lambda_{p}>\lambda_{p}$ et $\zeta \geqslant \frac{\Lambda_{p}}{p}$. La formule $(b)$ est encore vraie.

$\gamma) \Lambda_{p}>\lambda_{p}$ et $\zeta<\frac{\Lambda_{p}}{p}$. Ce cas est impossible. En effet, nous aurions 
alors $[\operatorname{voir}(f)]: \Lambda_{p}=\Lambda_{p-1}$, c'est-à-dire

$$
\zeta<\frac{\Lambda_{p-1}}{p}<\frac{\Lambda_{p-1}}{p-1} .
$$

Mais cette relation est en contradiction avec les formules (g) et $(a)$. Les formules (3.7) et (3.8) sont bien équivalentes.

Remarques. - a) Par les itérations (puissances) de la matrice $\mathfrak{D}$ nous obtenons la longueur des circuits qui peuvent contenir plusieurs circuits élémentaires (qui contiennent un sommet au plus une fois), cette longueur étant égale à la somme des longueurs des circuits élémentaires composants. Si toutes les longueurs des circuits sont négatives (ou nulles), alors la longueur d'un circuit n'est pas plus grande que celle d'une de ses composantes élémentaires. Mais cela n'est pas vrai pour les circuits du graphe $G^{*}$ qui sont tous d'une longueur positive. Il semble donc qu'il faille répéter les itérations de $\mathcal{D}$ au-delà de l'ordre $\nu^{\prime}$. Mais il est facile à voir que par une itération d'ordre $q>v^{\prime}$ on n'obtient pas une valeur $\varphi$ supérieure à celle que l'on a obtenu par les itérations d'ordre $\varphi^{\prime}$ au plus.

Considérons en effet un circuit qui se compose de circuits élémentaires possédant les ordres et les longueurs respectivement $n_{1}, l_{1} ; n_{2}, l_{2} ; \ldots$; $n_{k}, l_{k}\left(n_{i} \leqslant v^{\prime}\right)$. L'ordre du circuit considéré est $n^{\prime}=\Sigma n_{i}$ et sa longueur

$$
l^{\prime}=\Sigma l_{i}
$$

En considérant l'un des circuits élémentaires composants nous obtenons

$$
v_{i}=\frac{l_{i}}{n_{i}}
$$

alors que, en considérant le circuit entier, nous obtenons

$$
\iota^{\prime}=\frac{l^{\prime}}{n^{\prime}}=\frac{\Sigma l_{i}}{\Sigma n_{i}}
$$

Or on sait qu'on a la relation

$$
\frac{\Sigma l_{i}}{\Sigma n_{i}} \leqslant \max \frac{l_{i}}{n_{i}} \quad\left(l_{i}, n_{i} \geqslant 0\right),
$$

c'est-à-dire

$$
\varphi^{\prime} \leqslant \max _{i} v_{i}
$$

ce qui prouve notre affirmation; les itérations seront donc effectués seulement jusqu'à l'ordre v'.

b) L'algorithme proposé n'étant pas itératif comme le procédé dans [1], il comporte en général moins de calculs que celui-ci. 
Tandis qu'avec notre algorithme on doit effectuer $v^{\prime}-1$ multiplications de matrices, avec la méthode Cruon-Hervé on exécutera au cas le plus favorable $\nu^{\prime}-1$ multiplications et au cas le plus défavorable

$$
\frac{v^{\prime}\left(v^{\prime}+1\right)}{2}
$$

multiplications. Le rapport moyen entre les volumes de calcul nécessaires sera donc

$$
\varphi\left(\nu^{\prime}\right)=\frac{\nu^{\prime 2}+3 v^{\prime}-2}{4\left(\nu^{\prime}-1\right)}
$$

Le petit tableau suivant contient quelques valeurs de cette fonction :

\begin{tabular}{r|c}
$v^{\prime}$ & $\varphi\left(\nu^{\prime}\right)$ \\
\hline 2 & 2 \\
10 & 3,56 \\
50 & 13,5 \\
100 & 26,1
\end{tabular}

\section{BIBLIOGRAPHIE}

[1] R. Cruon et Ph. Hervé, Quelques résultats relatifs à une structure algébrique et son application au problème d'ordonnancement, Rev. Fr. Rech. Op. 9 (1965), $34,3-20$.

[2] L. Nemeti, Das Reihenfolgeproblem in der Fertigungsprogrammierung und Linearplanung mit logischen Bedingungen, Mathematica (Cluj), 6 (1964), 1, 87-99.

[3] F. Ra dó, Programare liniara cu conditii logice, Comunic. Acad. R.P.R. 13 (1963), 1039-1042.

[4] F. RADô, Un algorithme pour résoudre certains problèmes de programmation mathématique, Mathematica (Cluj), 6, (1964), 1, 105-116.

[5] B. Roy, Cheminement et connexité dans les graphes. Application aux problèmes d'ordonnancement, Metra, série spéciale, no 1-1962. 